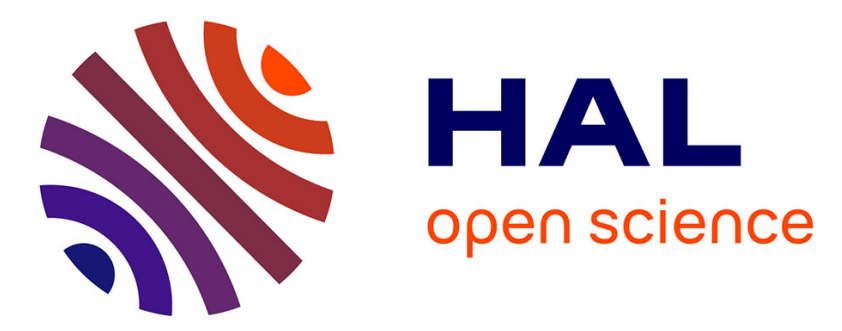

\title{
When targeted therapies alleviate the burden of TPE: The example of immune-mediated TTP
}

\author{
Adrien Picod, Paul Coppo
}

\section{To cite this version:}

Adrien Picod, Paul Coppo. When targeted therapies alleviate the burden of TPE: The example of immune-mediated TTP. Transfusion and Apheresis Science, 2019, 58, pp.273 - 277. 10.1016/j.transci.2019.04.012 . hal-03484463

\section{HAL Id: hal-03484463 https://hal.science/hal-03484463}

Submitted on 20 Dec 2021

HAL is a multi-disciplinary open access archive for the deposit and dissemination of scientific research documents, whether they are published or not. The documents may come from teaching and research institutions in France or abroad, or from public or private research centers.
L'archive ouverte pluridisciplinaire HAL, est destinée au dépôt et à la diffusion de documents scientifiques de niveau recherche, publiés ou non, émanant des établissements d'enseignement et de recherche français ou étrangers, des laboratoires publics ou privés.

\section{(ㄷ)(1) $\$$}

Distributed under a Creative Commons Attribution - NonCommerciall 4.0 International 
When targeted therapies alleviate the burden of TPE: the example of immune-mediated

\section{TTP}

Adrien Picod ${ }^{1}$, Paul Coppo ${ }^{1,2,3}$

1. Centre de Référence des MicroAngiopathies Thrombotiques, Paris, France

2. Service d'hématologie, Hôpital Saint-Antoine, Assistance publique - Hôpitaux de Paris,

France

3. Sorbonne Universités, Paris, France

Key words: Plasma exchange, plasmapheresis, thrombotic thrombocytopenic purpura, thrombotic microangiopathy, Moschcowitz syndrome, caplacizumab, ADAMTS13

\section{Corresponding author:}

Paul Coppo,

Centre de Référence des Microangiopathies thrombotiques (www.cnr-mat.fr)

Service d'hématologie

Hôpital Saint-Antoine

184 Rue du Faubourg Saint-Antoine

75012 Paris, France

Phone number: 00.33.1.49.28.26.21

Fax: 00.33.1.49.28.33.75

E-mail: paul.coppo@aphp.fr 


\section{List of abbreviations:}

TTP: Thrombotic thrombocytopenic purpura

ADAMTS13: a disintegrin and metalloprotease with thrombospondin type I repeats $-13^{\text {th }}$ member

vWF: von Willebrand Factor

LDH: Lactate dehydrogenase

IgG: Immunoglobulin G

\section{Abstract: 194}

Text word count: 2872

Table and figure: 3

References: 41 


\begin{abstract}
Thrombotic thrombocytopenic purpura is a rare and severe disease that manifests as a thrombotic microangiopathy with severe thrombocytopenia and variable multiorgan failure. The disease relies on a severe deficiency in a disintegrin and metalloprotease with thrombospondin type- 1 repeats, $13^{\text {th }}$ member (ADAMTS13), the von Willebrand factor (vWF) cleaving protease which can be either inherited (congenital TTP) or immune-mediated (iTTP). In iTTP, the therapeutic strategy has long relied on therapeutic plasma exchange alone which still represents the only way to deliver large amounts of ADAMTS13 without risking fluid overload. Yet, several therapeutic strategies have been developed in recent years and are about to transform the standard of care of iTTP. The immunosuppressive regimen now increasingly encompasses the administration of frontline rituximab to all patients. Moreover, the impressive results of the anti-vWF nanobody caplacizumab in phase 2 and 3 studies have recently prompted its approval by health authorities for the initial treatment of the disease. The increasing use of these highly effective targeted therapies should translate in a reduced need for therapeutic plasma exchange and an improvement in the prognosis of the disease. Nevertheless, and until the development of a recombinant ADAMTS13, this cornerstone therapy remains irreplaceable.
\end{abstract}




\section{Introduction}

Thrombotic thrombocytopenic purpura (TTP) is a life-threatening disease characterized by a severe deficiency in a distintegrin and metalloprotease with thrombospondin type 1 repeats, $13^{\text {th }}$ member (ADAMTS13), the von Willebrand factor (vWF)-cleaving protease. This deficiency leads to the accumulation of ultra-large vWF multimers both on the endothelial cells surface and into the circulation. By binding to platelets, these ultra-large vWF multimers prompt the formation of platelet-rich occlusive microthrombi in the microcirculation which subsequently cause ischemic organ dysfunction. The most affected organs are the central nervous system, the heart, and the digestive tract, but virtually all organs might be involved. A consumptive thrombocytopenia reflects microthrombi formation, whereas microangiopathic hemolytic anemia is related to red blood cells fragmentation on microthrombi, with increased shear stress in the microcirculation that maintains and amplifies these features. Accordingly, fragmented red blood cells, called schistocytes, are observed on patients' blood smear. Additionally, elevated serum lactate dehydrogenase (LDH) levels reflect hemolysis but primarily multivisceral ischemia.

The clinical picture of TTP usually associates mild fever (30 to 50\% of cases) and symptoms related to organs dysfunction. Neurological symptoms are observed in more than $50 \%$ of patients and range from headache to coma through transient and/or migratory focal deficiency, stupor and seizure. Life threatening hemorrhage related to severe thrombocytopenia might develop. The disease affects mostly adults with a female predominance (sex ratio $\approx 3 / 1$ ) and a maximum incidence between 30 and 50 years. ADAMTS13 deficiency might be congenital, especially in children and pregnant women, or more frequently acquired (immune-mediated) and related to the production of polyclonal autoantibodies against ADAMTS13, which is more common during adulthood. In this last situation, the disease might be isolated, or associated to conditions like human 
immunodeficiency virus infection, pregnancy, autoimmune diseases such as systemic lupus erythematosus, or antiplatelet agents use. The example of congenital TTP clearly illustrates that ADAMTS13 deficiency is necessary but not sufficient to induce a perceptible disease. Indeed, several conditions such as infection, inflammation or pregnancy act as triggering factors by activating endothelial cells and thus increasing vWF production. The imbalance between high concentrations of ultra-large vWF multimers and the low cleaving activity of ADAMTS13 either inherited or acquired then leads to the disease. Left untreated, iTTP is almost invariably fatal in the acute phase. Nowadays, the therapeutic regimen achieves survival rates as high as $90 \%$. However, iTTP is associated with relapses in up to $40 \%$ of survivors of a first episode, especially when ADAMTS13 activity remains persistently undetectable $(1,2)$.

TTP management as long been centered on the replenishment of the deficient ADAMTS13, either by the sole administration of donor's plasma, or by the combined extracorporeal removal of patient plasma and its replacement by donor's plasma, now referred as therapeutic plasma exchange (TPE). Given the autoimmune nature of iTTP, corticosteroids were then empirically added to the standard treatment. Yet, the recent development of therapies targeting the precise pathophysiological mechanisms of the disease, either autoantibodies production or interaction between platelets and vWF multimers, might change the therapeutic landscape of iTTP in the next few years. The purpose of this narrative review is to expose these recent advances in iTTP management and to detail how such therapies will translate into standard of care, and transforms the historical, TPE-centered therapy, of iTTP.

\section{Recent therapeutic advances in TTP management}

The success of rituximab in the treatment of various autoimmune conditions such as idiopathic thrombocytopenic purpura and autoimmune hemolytic anemia prompted its 
evaluation in iTTP, first for patients with a suboptimal response to treatment (i.e., patients experiencing an exacerbation or a refractory disease). In these reports, rituximab used as a salvage therapy resulted in higher remission rates (82 to 100\%) with faster responses to treatment and consequently fewer slow responders $(1,2)$. Interestingly, despite an often more severe initial presentation, patients who received rituximab experienced fewer 2-year relapses that occurred later in accordance with a prolonged immunosuppression. Given these encouraging results, a randomized trial was initiated by the Transfusion Medicine/Hemostasis Clinical Trials Network to evaluate the efficacy of systematic administration of rituximab as part of the frontline management of iTTP (3). Unfortunately, because of a very low rate of inclusion, this study was prematurely terminated and the only evidence available comes from an open-label trial from the South East England TTP study group (4). In this study, the early ( $\leq 3$ days from admission) administration of rituximab in addition to a standard therapeutic regimen including TPE and corticosteroids, resulted in a high response rate with fewer relapses. Additionally, there was a trend toward a shorter duration of TPE therapy for rituximab-treated patients when compared with historical controls. Interestingly, a subsequent study from the same group compared the outcome of patients who received rituximab early $(\leq$ 3 days, most of these patients having been included in the aforementioned trial) vs late (> 3 days) and confirmed the benefit of an early administration with regards to the time to remission (median, 12 days $v s 20$ days, respectively, $\mathrm{p}<0.001$ ), the number of TPE procedures (median, 16 vs 24, respectively, $\mathrm{p}=0.03$ ) and the length of hospital stay (median, 16 days $v s$ 23 days, respectively, $\mathrm{p}=0.01)(5)$.

More recently, therapies targeting the interaction between platelets and vWF multimers have been developed with the aim to prevent the formation of microthrombi and subsequent microcirculation occlusion and ischemic organ damage. Caplacizumab (Cablivi ${ }^{\mathrm{TM}}$, 
Ablynx, a Sanofi Company), formerly known as ALX-0081 (intravenous administration) and ALX-0681 (subcutaneous administration), is a bivalent nanobody, i.e., a humanized singlevariable domain antibody derived from the homodimeric heavy-chain antibodies naturally occurring in camelids (6). This small format of $28 \mathrm{kDa}$ offers several theoretical advantages compared to classically manufactured antibodies: rapid distribution and rapid clearance in order to allow both a rapid onset of action and a limitation of potential toxicity; specific, high affinity, and irreversible binding to the target and direct action without Fc-mediated recruitment of cellular effectors or complement activation, preventing potential adverse effects. Caplacizumab targets the A1 domain of ultra-large vWF multimers and thus blocks its interaction and binding to the platelet glycoprotein Ib-IX-V receptor, which is the critical first step of platelet aggregation. The drug was first developed as an antithrombotic agent in the context of myocardial infarction, but greater interest has soon been paid to its therapeutic potential in TTP, and development for the first indication has since been discontinued. Indeed, in the absence of interaction between platelets and $\mathrm{vWF}$, the formation of occlusive microthrombi can be prevented. In a pre-clinical baboon model of iTTP obtained by the administration of a neutralizing anti-ADAMTS13 antibody, the daily administration of caplacizumab allowed a rapid ( $<1$ day) and profound inhibition of vWF activity as measured by ristocetin cofactor activity which recovered seven days after treatment cessation. This inhibition of vWF activity translated into clinical efficacy with a rapid recovery of platelet count and a decrease in LDH level along with a trend toward the resolution of the microangiopathic hemolytic anemia in treated animals compared to untreated controls. However, at postmortem analysis, the proportion of occluded vessels did not differ between groups, suggesting that caplacizumab was not able to dissolve previously formed microthrombi (7). 
Preliminary data showed that effective, steady-state concentration was reached in humans following once daily subcutaneous administration of caplacizumab 10mg and this therapeutic schedule was then adopted for clinical trials with the addition of one intravenous dose prior to the first TPE, in order to achieve an immediate onset of action. So far, caplacizumab has been evaluated in two randomized controlled trials (Table 1). In the phase II TITAN trial, the addition of caplacizumab to standard treatment and for 30 days after the last TPE resulted in a significant reduction in the time to response, defined as the time to confirmed normalization of the platelet count (median 2.97 days, vs. 4.79 days in placebo) (8). Two deaths occurred in the placebo group vs none in patients treated with caplacizumab. However, relapses were more frequent in the caplacizumab group with seven patients relapsing within 10 days after caplacizumab had been stopped. In these seven patients, ADAMTS13 activity was persistently less than 10\%, suggesting persistent auto-immune inhibition of the enzyme. These results were then confirmed in a phase III randomized clinical trial (9). Again, the addition of caplacizumab to standard treatment resulted in a significant reduction in the time to platelet count response (platelet count normalization rate $1.55,95 \%$ CI $1.10-2.20, \mathrm{p}<0.01)$. Additionally, there was a significant reduction in a composite secondary endpoint of iTTP-related death, recurrence of iTTP or $\geq 1$ major thromboembolic event during the study treatment period compared with placebo (12.5\% vs $49.3 \%$, respectively, $\mathrm{p}<0.0001)$. Unlike for the phase II trial, investigators were encouraged to extend the blinded treatment for a maximum of 4 weeks along with optimization of the immunosuppression regimen if there was evidence of an ongoing disease, that is, a persistently suppressed ADAMTS13 activity. This strategy translated into a reduced rate of iTTP recurrence (including exacerbation and relapse) in the caplacizumab group $(12.5 \% \mathrm{vs}$ $38.4 \%$, respectively, $\mathrm{p}<0.001)$. In accordance to its mechanism of action, side effects associated with the use of caplacizumab in both studies were mainly represented by a greater 
occurrence of muco-cutaneous bleeding such as epistaxis and gingival bleeding (Table 2). However, these events were mild and rarely clinically significant, with no death attributable to treatment. Long term efficacy and safety of caplacizumab is now being evaluated in all patients who completed the HERCULES trial in a phase IIIb prospective follow-up study (Post-HERCULES trial, NCT02878603).

Another major therapeutic achievement in the field of TTP is the development of a recombinant human ADAMTS13 (rhADAMTS13) (BAX930, Baxalta, part of Shire). In a phase I study conducted in patients suffering from congenital TTP, the administration of rhADAMTS13 led to a dose-dependent detection of vWF-cleavage products as well as a trend toward LDH decrease and platelet count improvement. Additionally, administration of the recombinant enzyme was well tolerated, and importantly, no patient developed detectable neutralizing anti-ADAMTS13 antibodies (10). A phase III trial is currently evaluating the efficacy of prophylactic administration of rhADAMTS13 to prevent acute episodes in congenital TTP (NCT03393975). In the next future, this therapeutic agent could also show efficacy in iTTP. Indeed, it has been shown that the addition of rhADAMTS13 to patients' plasma was able to restore ADAMTS13 activity with a linear correlation between the inhibitory titer and the requirement of rhADAMTS13 (11).

\section{Present and future of TPE management}

TPE still remains the centerpiece iTTP treatment. Its effectiveness was suggested by the mention of transfusion exchange techniques as early as the description of the disease by Moschcowitz in 1924 (12). Several variants of transfusion exchange have subsequently been used and remarkably, only the techniques including plasma infusion proved efficacy. This important clinical observation had two important consequences: first, it led to the conclusion 
that a deficient factor was provided by plasma (which paved the way for the identification of ADAMTS13 $(13,14))$; second, it defined TPE as the reference treatment of iTTP for decades (15). The superior efficacy of TPE compared to the sole infusion of plasma in early studies probably relies on the difference in volume (3 times higher for TPE) and therefore on the quantity of ADAMTS13 infused (16). The supply of large volumes of plasma (25 to $30 \mathrm{~mL} / \mathrm{kg} /$ day) can constitute a standby treatment if TPE is not available immediately, although such treatment rapidly exposes patients to fluid overload $(17,18)$.

TPE should be started as soon as possible because any delay worsens the prognosis (19). TPE can be performed either using centrifugation or filtration depending on team's experience and/or availability of the corresponding device. It is common to start the treatment by exchanging $1.5 \mathrm{x}$ the estimated plasma volume and then reducing the exchanged volume to 1.0x. The replacement fluid should be plasma only since other fluids do not bring ADAMTS13. However, some teams used 50\% albumin 4-5\% and 50\% plasma with apparently comparable reported results (20). Various preparations of plasma have been tested based on the rationale of varying content in vWF multimers, while ADAMTS13 activity remains constant (21). To date, there is no clearly demonstrated superiority between quarantine fresh frozen plasma, amotosalen-inactivated plasma and solvent-detergent inactivated plasma. Of note, methylene-blue-photoinactivated plasma was associated with more TPE sessions vs fresh frozen plasma to achieve durable remission (22-24); moreover, its use has been discontinued in several countries as side effects were more prevalent. In patients who do not improve with standard treatment, twice-daily TPE has been reported as an available salvage strategy $(25,26)$. TPE should be continued until remission, as defined by platelet recovery (platelet count > 150G/L for more than 48h), LDH decrease and clinical improvement (27). Historically, TPE used to be tapered over several weeks to prevent (and/or to control more rapidly) an exacerbation of the disease after cessation. However, the 
increasing use of concomitant immunosuppressive strategies as well as the use of caplacizumab in the next future should allow interrupting TPE abruptly without any detrimental consequence. The accurate monitoring of ADAMTS13 activity provides confidence in this attitude when a recovery of ADAMTS13 activity is observed (at least > $20 \%$ activity). Conversely, a persistently suppressed (i.e. < 10\%) ADAMTS13 activity, although it does not necessarily preclude TPE cessation -especially under the coverage of anti-vWF therapy-, should plead for an optimization of immunosuppression.

The benefit of TPE usually relies on the elimination of one or several toxic factors and/or on the supplementation in one or several deficient factors. In the specific context of iTTP, the clinical benefit of TPE could result from three actions: the removal of autoantibodies directed against ADAMTS13; the removal of ultra-large vWF multimers; and most importantly, the supplementation in ADAMTS13 without risking fluid overload. However, the last statement may be the most clinically relevant. Indeed, autoantibodies against ADAMTS13 are mainly of the immunoglobulin $\mathrm{G}(\operatorname{IgG})$ isotype $(13,14,28,29)$, which intravascular distribution only represents $45 \%$ of the total pool (30). Thus, each decrease in IgG concentration after a TPE session is invariably followed by a rebound increase relying both on the new equilibrium between intravascular and extravascular pool of $\mathrm{IgG}$, and on the rate of de novo synthetized IgG (30). Moreover, high autoantibodies titers can persist despite intensive TPE therapy (31-33). Lastly, removal of ultra-large vWF multimers by TPE, albeit partially effective, is counterbalanced by a continuous production and it is likely that only the administration of a sufficient quantity of ADAMTS13 is able to reduce platelet clumping by cleaving ultra-large vWF multimers.

Because of the need to rapidly suppress the production of anti-ADAMTS13 antibodies, an immunosuppressive strategy should be initiated as soon as possible. 
Historically, and despite the lack of strong clinical evidence, corticosteroids have been empirically administered to achieve this goal. Corticosteroids are usually used at doses of 1 to $1.5 \mathrm{mg} / \mathrm{kg} /$ day of prednisone or equivalent although higher doses might be of interest. Iin a multicentric randomized open-label trial, 60 patients were randomized to receive either methylprednisolone $1 \mathrm{mg} / \mathrm{kg} /$ day or $10 \mathrm{mg} / \mathrm{kg} /$ day for 3 days, followed by $2.5 \mathrm{mg} / \mathrm{kg} /$ day in addition to TPE. After 23 days of treatment, a significant reduction in the proportion of patients refractory to therapy was noted in the high-dose group (23.4 vs. 53.4, respectively, p $=0.03)(34)$.

Following this line of thought, frontline adjuvant rituximab should now be administered to all patients to achieve rapid, efficient and durable immunosuppression and prevent short-term relapses (Figure 1) (Coppo et al, Res Pract Thromb Haemost 2019, in press). Furthermore, it appears that rituximab is well tolerated, with no increase in the overall infections rate (2). Moreover, this strategy could be cost-effective by saving a full cumbersome management in case of relapse (35). However, the optimal dose and timing of rituximab infusions remains to be determined. Indeed, most studies used a 4-weeks regimen of once-weekly $375 \mathrm{mg} / \mathrm{m}^{2}$ infusion. A more intensive regimen has been evaluated considering the fact that a substantial amount of rituximab was eliminated during TPE; however kinetics of B-cell depletion in this work proved to be similar to previous studies (36). Conversely, lower doses of rituximab (100mg/week for 4 weeks or $375 \mathrm{mg} / \mathrm{m}^{2} \times 2$ to 3 infusions) showed a similar efficacy (37-40).

Several other immunosuppressive treatments have been used as salvage therapies in refractory patients, including mainly cyclophosphamide, vincristine, cyclosporine, or splenectomy and more recently anti-plasma cell agents (Coppo et al, Res Pract Thromb Haemost 2019, in press) $(1,41)$. However, their role in the modern treatment strategy including TPE, caplacizumab, corticosteroids, and rituximab, needs to be redefined. 


\section{Conclusion}

TPE still represents the cornerstone of iTTP management. It not only allowed a dramatic improvement in the previously dismal prognosis of the disease, but it also helped for a better understanding of the underlying mechanisms of the disease. In addition to the historical treatment consisting of TPE and corticosteroids, frontline rituximab and caplacizumab should now be part of the standard of care as they should further improve survival by preventing early death, exacerbations of the disease, as well as delayed responses and short term relapse. The subsequent reduction in the number of TPE sessions should lead to less treatment-related complications; further studies should also assess the costeffectiveness of such new strategy. In the next future, one could speculate that the burden of TPE in the management of iTTP should decrease even more with the availability of rhADAMTS13. No doubt that the history of TTP treatment will require additional chapters to be written in the forthcoming years.

\section{Declaration of interest:}

AP have no competing interest. PC is a member of the advisory board for Alexion, Octapharma, Shire and Ablynx part of Sanofi-Genzyme. 


\section{References:}

1. Kremer Hovinga JA, Coppo P, Lämmle B, Moake JL, Miyata T, Vanhoorelbeke K. Thrombotic thrombocytopenic purpura. Nat Rev Dis Primer. 2017 Apr 6;3:17021.

2. Joly BS, Coppo P, Veyradier A. Thrombotic thrombocytopenic purpura. Blood. 2017 May 25;129(21):2836-46.

3. George JN, Woodson RD, Kiss JE, Kojouri K, Vesely SK. Rituximab therapy for thrombotic thrombocytopenic purpura: A proposed study of the Transfusion Medicine/Hemostasis Clinical Trials Network with a systematic review of rituximab therapy for immune-mediated disorders. J Clin Apheresis. 2006 Apr;21(1):49-56.

4. Scully M, McDonald V, Cavenagh J, Hunt BJ, Longair I, Cohen H, et al. A phase 2 study of the safety and efficacy of rituximab with plasma exchange in acute acquired thrombotic thrombocytopenic purpura. Blood. 2011 Aug 18;118(7):1746-53.

5. Westwood J-P, Webster H, McGuckin S, McDonald V, Machin SJ, Scully M. Rituximab for thrombotic thrombocytopenic purpura: benefit of early administration during acute episodes and use of prophylaxis to prevent relapse. J Thromb Haemost. 2013 Mar;11(3):481-90.

6. Hamers-Casterman C, Atarhouch T, Muyldermans S, Robinson G, Hammers C, Songa EB, et al. Naturally occurring antibodies devoid of light chains. Nature. 1993 Jun;363(6428):446-8.

7. Callewaert F, Roodt J, Ulrichts H, Stohr T, van Rensburg WJ, Lamprecht S, et al. Evaluation of efficacy and safety of the anti-VWF Nanobody ALX-0681 in a preclinical baboon model of acquired thrombotic thrombocytopenic purpura. Blood. 2012 Oct 25;120(17):3603-10.

8. Peyvandi F, Scully M, Kremer Hovinga JA, Cataland S, Knöbl P, Wu H, et al. Caplacizumab for Acquired Thrombotic Thrombocytopenic Purpura. N Engl J Med. 2016 Feb 11;374(6):511-22.

9. Scully M, Cataland SR, Peyvandi F, Coppo P, Knöbl P, Kremer Hovinga JA, et al. Caplacizumab Treatment for Acquired Thrombotic Thrombocytopenic Purpura. N Engl J Med. 2019 Jan 9;

10. Scully M, Knöbl P, Kentouche K, Rice L, Windyga J, Schneppenheim R, et al. Recombinant ADAMTS-13: first-in-human pharmacokinetics and safety in congenital thrombotic thrombocytopenic purpura. Blood. 2017 Nov 9;130(19):2055-63.

11. Plaimauer B, Kremer Hovinga JA, Juno C, Wolfsegger MJ, Skalicky S, Schmidt M, et al. Recombinant ADAMTS13 normalizes von Willebrand factor-cleaving activity in plasma of acquired TTP patients by overriding inhibitory antibodies: rADAMTS13 overrides inhibitors in TTP plasma. J Thromb Haemost. 2011 May;9(5):936-44.

12. Moschcowitz E. An acute febrile pleiochromic anemia with hyaline thrombosis of the terminal arterioles and capillaries: an unde- scribed disease. Arch Intern Med. $1925 \mathrm{Jul}$ $1 ; 36(1): 89$. 
13. Furlan M, Robles R, Galbusera M, Remuzzi G, Kyrle PA, Brenner B, et al. von Willebrand Factor-Cleaving Protease in Thrombotic Thrombocytopenic Purpura and the Hemolytic-Uremic Syndrome. N Engl J Med. 1998 Nov 26;339(22):1578-84.

14. Tsai H-M, Lian EC-Y. Antibodies to von Willebrand Factor-Cleaving Protease in Acute Thrombotic Thrombocytopenic Purpura. N Engl J Med. 1998 Nov 26;339(22):1585-94.

15. Byrnes JJ, Khurana M. Treatment of Thrombotic Thrombocytopenic Purpura with Plasma. N Engl J Med. 1977 Dec 22;297(25):1386-9.

16. Rock GA, Shumak KH, Buskard NA, Blanchette VS, Kelton JG, Nair RC, et al. Comparison of Plasma Exchange with Plasma Infusion in the Treatment of Thrombotic Thrombocytopenic Purpura. N Engl J Med. 1991 Aug 8;325(6):393-7.

17. Novitzky N, Jacobs P, Rosenstrauch W. The treatment of thrombotic thrombocytopenic purpura: plasma infusion or exchange? Br J Haematol. 1994 Jun;87(2):317-20.

18. Coppo P, Bussel A, Charrier S, Adrie C, Galicier L, Boulanger E, et al. High-dose plasma infusion versus plasma exchange as early treatment of thrombotic thrombocytopenic purpura/hemolytic-uremic syndrome. Medicine (Baltimore). 2003 Jan;82(1):27-38.

19. Pereira A, Mazzara R, Monteagudo J, Sanz C, Puig L, Martínez A, et al. Thrombotic thrombocytopenic purpura/hemolytic uremic syndrome: a multivariate analysis of factors predicting the response to plasma exchange. Ann Hematol. 1995 Jun;70(6):319_ 23.

20. O'Brien KL, Price TH, Howell C, Delaney M. The use of $50 \%$ albumin/plasma replacement fluid in therapeutic plasma exchange for thrombotic thrombocytopenic purpura: Fifty Percent Albumin/Plasma Fluid for TPE in TTP. J Clin Apheresis. 2013 Dec;28(6):416-21.

21. Hacquard M, Lecompte T, Belcour B, Geschier C, Jacquot C, Jacquot E, et al. Evaluation of the hemostatic potential including thrombin generation of three different therapeutic pathogen-reduced plasmas: Hemostatic potential of therapeutic plasma. Vox Sang. 2012 May;102(4):354-61.

22. Alvarez-Larran A, Del Rio J, Ramirez C, Albo C, Pena F, Campos A, et al. Methylene blue-photoinactivated plasma vs. fresh-frozen plasma as replacement fluid for plasma exchange in thrombotic thrombocytopenic purpura. Vox Sang. 2004 May;86(4):246-51.

23. del Río-Garma J, Alvarez-Larrán A, Martínez C, Muncunill J, Castellà D, de la Rubia J, et al. Methylene blue-photoinactivated plasma versus quarantine fresh frozen plasma in thrombotic thrombocytopenic purpura: a multicentric, prospective cohort study. Br J Haematol. 2008 Oct;143(1):39-45.

24. de la Rubia J, Arriaga F, Linares D, Larrea L, Carpio N, Marty ML, et al. Role of methylene blue-treated or fresh-frozen plasma in the response to plasma exchange in patients with thrombotic thrombocytopenic purpura. Br J Haematol. 2001 Sep;114(3):721-3. 
25. Soucemarianadin M, Benhamou Y, Delmas Y, Pichereau C, Maury E, Pène F, et al. Twice-daily therapeutical plasma exchange-based salvage therapy in severe autoimmune thrombotic thrombocytopenic purpura: the French TMA Reference Center experience. Eur J Haematol. 2016 Aug;97(2):183-91.

26. Nguyen L, Li X, Duvall D, Terrell DR, Vesely SK, George JN. Twice-daily plasma exchange for patients with refractory thrombotic thrombocytopenic purpura: the experience of the Oklahoma Registry, 1989 through 2006. Transfusion (Paris). 2007 Nov 20;48(2):349-57.

27. Scully M, Cataland S, Coppo P, de la Rubia J, Friedman KD, Kremer Hovinga J, et al. Consensus on the standardization of terminology in thrombotic thrombocytopenic purpura and related thrombotic microangiopathies. J Thromb Haemost. 2017 Feb;15(2):312-22.

28. Rieger M. ADAMTS13 autoantibodies in patients with thrombotic microangiopathies and other immunomediated diseases. Blood. 2005 Aug 15;106(4):1262-7.

29. Ferrari S, Scheiflinger F, Rieger M, Mudde G, Wolf M, Coppo P, et al. Prognostic value of anti-ADAMTS13 antibodies features (Ig isotype, titer and inhibitory effect) in a cohort of 35 adult French patients undergoing a first episode of thrombotic microangiopathy with an undetectable ADAMTS13 activity. Blood. 2006 Dec 12;

30. Kaplan AA. Therapeutic plasma exchange: A technical and operational review: Therapeutic Plasma Exchange. J Clin Apheresis. 2013 Feb;28(1):3-10.

31. Furlan M, Robles R, Morselli B, Sandoz P, Lämmle B. Recovery and Half-Life of von Willebrand Factor-Cleaving Protease after Plasma Therapy in Patients with Thrombotic Thrombocytopenic Purpura. Thromb Haemost. 1999;81(01):8-13.

32. Zheng XL. Effect of plasma exchange on plasma ADAMTS13 metalloprotease activity, inhibitor level, and clinical outcome in patients with idiopathic and nonidiopathic thrombotic thrombocytopenic purpura. Blood. 2004 Jun 1;103(11):4043-9.

33. Mannucci PM, Bohm M, Scharrer I, Scheiflinger F. Patterns of changes of antiADAMTS13 after plasma exchange. J Thromb Haemost. 2006 Jun;4(6):1405-6.

34. The Italian TTP Study Group, Balduini CL, Gugliotta L, Luppi M, Laurenti L, Klersy C, et al. High versus standard dose methylprednisolone in the acute phase of idiopathic thrombotic thrombocytopenic purpura: a randomized study. Ann Hematol. 2010 Jun;89(6):591-6.

35. Sadler JE. Von Willebrand factor, ADAMTS13, and thrombotic thrombocytopenic purpura. Blood. 2008 Feb 26;112(1):11-8.

36. Froissart A, Buffet M, Veyradier A, Poullin P, Provôt F, Malot S, et al. Efficacy and safety of first-line rituximab in severe, acquired thrombotic thrombocytopenic purpura with a suboptimal response to plasma exchange. Experience of the French Thrombotic Microangiopathies Reference Center: Crit Care Med. 2012 Jan;40(1):104-11.

37. Pequeño-Luévano M, Villarreal-Martínez L, Jaime-Pérez JC, Gómez-de-León A, CantúRodríguez OG, González-Llano O, et al. Low-dose rituximab for the treatment of acute 
thrombotic thrombocytopenic purpura: Report of four cases. Hematology. 2013 Jul;18(4):233-6.

38. Vazquez-Mellado A, Pequeño-Luévano M, Cantu-Rodriguez OG, Villarreal-Martínez L, Jaime-Pérez JC, Gomez-De-Leon A, et al. More about low-dose rituximab and plasma exchange as front-line therapy for patients with thrombotic thrombocytopenic purpura. Hematology. 2016 May 27;21(5):311-6.

39. Benhamou Y, Paintaud G, Azoulay E, Poullin P, Galicier L, Desvignes C, et al. Efficacy of a rituximab regimen based on $\mathrm{B}$ cell depletion in thrombotic thrombocytopenic purpura with suboptimal response to standard treatment: Results of a phase II, multicenter noncomparative study: B cell driven rituximab therapy in TTP. Am J Hematol. 2016 Dec;91(12):1246-51.

40. Zwicker J. Efficacy of Adjuvant Low Dose Rituximab and Plasma Exchange for Acquired TTP with Severe ADAMTS13 Deficiency - Results of the ART Study. In ASH; 2018.

41. Patriquin CJ, Thomas MR, Dutt T, McGuckin S, Blombery PA, Cranfield T, et al. Bortezomib in the treatment of refractory thrombotic thrombocytopenic purpura. Br J Haematol. 2016 Jun;173(5):779-85. 
Figure 1. The three axes of iTTP management in the acute phase.

* Other rituximab administration regimens are currently under investigation.

TPE: Therapeutic plasma exchange. LDH: Lactate dehydrogenase. vWF: von Willebrand factor. 
Table 1. First-line treatment with caplacizumab vs placebo in iTTP: data from TITAN and HERCULES randomized controlled trials.

\begin{tabular}{|c|c|c|c|c|c|c|c|c|c|}
\hline Reference & $\begin{array}{l}\text { Number of patients } \\
\text { Caplacizumab vs placebo }\end{array}$ & $\begin{array}{l}\text { Mean age }(\mathbf{y}) \\
\text { (range) }\end{array}$ & $\begin{array}{l}\text { Female } \\
\mathbf{N}(\%)\end{array}$ & CR \% & Recurrence* \% & Exacerbation \% & Relapse \% & $\begin{array}{l}\text { Mortality in the } \\
\text { acute phase \% }\end{array}$ & $\begin{array}{l}\text { Mean TPE } \\
\text { duration (d) }\end{array}$ \\
\hline TITAN (8) & 75 (36 vs 39) & $42(19-72)$ & $44(59)$ & 81 vs 46 & 38.9 vs 38.4 & 8.3 vs 28.2 & 30.6 vs $7.7^{\$}$ & 0 vs 5.1 & 5.9 vs 7.9 \\
\hline HERCULES (9) & 145 (72 vs 73) & $46(18-79)$ & $100(69)$ & NA & 12.5 vs 38.3 & 4.2 vs 38.3 & 8.3 vs $0^{f}$ & 0 vs 4.1 & 5.8 vs 9.4 \\
\hline
\end{tabular}

CR: complete remission; y: years; d: days; TPE: therapeutic plasma exchange. *recurrence was defined as exacerbation and/or relapse; ${ }^{\$} 12$

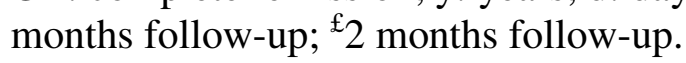


Table 2. Adverse events in patients treated with caplacizumab vs placebo (adapted from $(8,9)$ ).

\begin{tabular}{|l|l|l|}
\hline Adverse event & $\begin{array}{l}\text { TITAN } \\
\text { Caplacizumab vs placebo }\end{array}$ & $\begin{array}{l}\text { HERCULES } \\
\text { Caplacizumab vs placebo }\end{array}$ \\
\hline Bleeding & 54.2 vs 37.8 & 64.8 vs 47.9 \\
\hline Gingival bleeding & 14.3 vs 5.4 & 18.3 vs 1.4 \\
\hline Epistaxis & 31.4 vs 10.8 & 32.4 vs 2.7 \\
\hline Hematuria & 0 vs 2.7 & 7.0 vs 2.7 \\
\hline Catheter site hemorrhage & NA & 7.0 vs 6.8 \\
\hline Subarachnoid hemorrhage & 2.9 vs 0 & 1.4 vs 0 \\
\hline Headache & 34.3 vs 27 & 22.5 vs 8.2 \\
\hline Pyrexia & 17.1 vs 16.2 & 14.1 vs 8.2 \\
\hline Myalgia & 20.0 vs 2.7 & NA \\
\hline Urticaria & NA & 16.9 vs 6.8 \\
\hline
\end{tabular}

NA: not available. Data are provided as percent. 


\section{ADAMTS13 supplementation}

- Daily TPE with 1.5 plasma volume

- Until clinical improvement + platelet count $>150 \mathrm{G} / \mathrm{L}$ for two days + decrease in LDH level

- In unresponsive patients, consider twice daily TPE

- Corticosteroids 1 to $1.5 \mathrm{mg} / \mathrm{kg} \pm$ boluses Taper when ADAMTS13 activity recovers (e.g. $>20 \%$ )

- Rituximab: $375 \mathrm{mg} / \mathrm{m}^{2}$ IV x3 to 4 within 2 to 3 weeks*
Inhibition of platelets-vWF interaction
- Caplacizumab: $10 \mathrm{mg}$ IV followed by $10 \mathrm{mg}$ SC daily

- Until ADAMTS13 activity recovers (e.g. $>20 \%$ ) 